\title{
Application Research Progress of Neuromodulation Technology in the Treatment of Freezing Gait in Parkinson's Disease
}

\author{
Guolin Shi, Ninghui Zhao", Tingting Wu \\ The Second Affiliated Hospital of Kunming Medical University, Kunming 650101, Yunnan, China \\ Email: 282077116@qq.com
}

\begin{abstract}
Freezing gait is a common clinical symptom in patients with Parkinson's disease. It tends to get worse with the progress of the disease, causing the patients to fall easily, which is one of the important reasons for the loss of independent living ability. The treatment of frozen gait is very complicated, and drug treatment has no obvious effect. Neuromodulation technology is currently an effective method for clinical treatment of frozen gait in Parkinson's disease. This technology mainly uses implantable devices and non-implantable devices, and uses electrical or chemical methods to correct frozen gait, which helps to improve the quality of life of patients with Parkinson's disease. This article focuses on the application research of neuromodulation technology in the treatment of freezing gait in Parkinson's disease.
\end{abstract}

Keywords: Parkinson's disease, freezing gait, neuromodulation technology

\section{Introduction}

Freezing gait is common in the middle and late stages of Parkinson's disease, leading to a decline in the patients' ability to live independently and increasing the burden on the family and society. Therefore, how to treat frozen gait, improve the living ability of patients with Parkinson's disease and reduce the burden on family and society has become an important topic of clinical research. Neuromodulation technology is a new type of clinical treatment technology, and currently shows good application effects in the clinical treatment of movement disorders, mental disorders and intractable pain ${ }^{[1]}$. With its popularization and application in clinical treatment, it is found that this technology has a better effect in the treatment of freezing gait in Parkinson's disease, showing unique therapeutic advantages.

\section{The mechanism of frozen gait in Parkinson's disease}

Freezing gait refers to a brief, sudden step termination or reduction during walking. It is a non-specific symptom that can be induced by a variety of diseases, such as primary freezing gait, dyskinesia, hydrocephalus, Parkinson's disease, and multiple system atrophy. Studies have pointed out that about $80 \%$ of patients with advanced Parkinson's disease are accompanied by freezing gait symptoms ${ }^{[2]}$.

Due to the different clinical manifestations of patients with frozen gait, it is difficult to evaluate the severity of frozen gait. At present, most scholars use various scales for evaluation, but they are subjectively affected, so they cannot be widely used. Some scholars improve the diagnostic accuracy of frozen gait by inducing experiments to perform tasks jointly, but there are some problems in practical applications that cannot be widely used ${ }^{[3]}$. At present, some research institutes propose the use of virtual technology for gait simulation to evaluate the condition of frozen gait in patients with Parkinson's disease. Some scholars have also proposed using wearable devices and sensors to obtain data such as the height of the feet and walking speed of patients with Parkinson's disease, so as to determine whether the patient has frozen gait

${ }^{[4]}$. With the continuous innovation of modern science and technology, the emergence of various detection technologies has made the detection rate of frozen gait of patients with Parkinson's disease continue to increase.

\section{The application of neuromodulation technology in the clinical treatment of frozen gait in Parkinson's disease}

Neuromodulation technology first appeared in the late 1970s. It is a technology that has developed relatively rapidly in biomedicine and engineering medical technology in recent years, providing a new direction for the treatment of many diseases. Nerve regulation technology mainly affects the function of the nervous system, but has a greater impact on all tissues and organs of the body. This technology mainly uses implantable devices and non-implantable devices to improve the activity of the central nervous system, peripheral nervous system and autonomic nervous system through electrical 
stimulation or drugs, further improve the clinical symptoms of patients and improve the quality of life of patients ${ }^{[5]}$. At present, there are many types of neuromodulation technologies in clinical application, which can be divided into invasive neuromodulation and non-invasive neuromodulation according to the characteristics of the operation. And neuromodulation technology has the advantages of targeted therapy, good controllability, and high safety, and has good therapeutic effects for a variety of systemic diseases, such as epilepsy, Alzheimer's disease and Parkinson's disease ${ }^{[6]}$. As a nerve treatment method, neuromodulation technology can not only stimulate target neurons and peripheral nerve nuclei to activate nerve activity, adjust the sensitivity of synapses, and promote nerve function to return to normal, but also can stimulate the cerebral cortex and restore nerve action Potential affects brain metabolism, improves brain perfusion, accelerates the recovery of nerve function, thereby effectively improving the symptoms of abnormal gait in patients with Parkinson's disease and improving the quality of life of patients.

\subsection{Deep brain stimulation technology}

At present, clinical studies have not yet clarified the specific mechanism of freezing gait in patients with Parkinson's disease, but most scholars believe that basal ganglia neural circuit dysfunction plays an important role in the mechanism of this symptom. However, in clinical studies, it was found that some patients treated with dopamine drugs had no obvious effect. It can be seen that this mechanism is not the only mechanism that causes freezing of gait. Deep brain electrical stimulation technology is a stereotactic technology, which mainly uses stereotactic computer based on the results of head $\mathrm{CT}$ and MRI examinations and microelectrode recordings. Electrodes are placed at specific targets and pulsed electrical signals are released to stimulate peripheral neurons. And nerve nuclei, thereby regulating neurotransmitters and activating axons, thereby improving the patient's nerve function and achieving the effect of alleviating freezing gait. Some scholars believe that the frozen gait of patients with Parkinson's disease is closely related to cognitive dysfunction, which may be due to abnormal nerve conduction pathways in motor, cognition and emotion. Frozen gait mainly occurs in unilateral lower limbs in the early stage, and develops frequently in bilateral lower limbs as the disease progresses. The patient feels as if his legs are frozen while walking, which lasts for several seconds to several minutes. According to the patient's gait performance, the frozen gait can be divided into the small-step prolapsed type, the original tremor type, and the completely unable to move. According to the sensitivity of patients to dopamine drugs, freezing gait can be divided into dopamineresponsive, dopamine-resistant and dopamine-inducible. Studies have found that deep brain stimulation technology has a better therapeutic effect on various types of frozen gait. At present, the main targets of this technology to treat frozen gait are mostly the basal ganglia and midbrain motor areas. Among them, the subthalamic nucleus and the medial part of the globus pallidus have a higher incidence. By adjusting the frequency of electrical stimulation of the brain nuclei, it can not only effectively improve the patient's tremor, muscle rigidity and freezing gait symptoms, but also improve the patient's midline symptoms ${ }^{[7]}$. The specific mechanism of action of deep brain stimulation technology is not yet fully understood. At present, clinically, it is mainly to inhibit the activity of the stimulated nucleus by stimulating the target to change the abnormal output pattern of the basal ganglia, and produce a more regular activity effect in the downstream structure, so as to achieve the effect of changing the abnormal action.

\subsection{Transcranial magnetic stimulation technology}

Transcranial magnetic stimulation technology is a non-invasive treatment technique. It mainly uses the principle of Faraday electromagnetic induction to place a layer of energized coil on the surface of the scalp to change the action potential through the induced current generated by the magnetic field through the cerebral cortex, causing a series of physiological and biochemical changes in the brain tissue, thereby improving the brain metabolism. Clinical studies have found that low-frequency stimulation can effectively inhibit the excitability of the central brain system, thereby reducing blood flow. High-frequency stimulation can improve the excitability of the central brain system, accelerate the cerebral blood circulation and metabolism ${ }^{[8]}$. However, there are currently clinical controversies regarding this therapy, and it is still necessary to expand the sample size for further research and observation.

\subsection{Transcranial direct current stimulation technology}

Transcranial direct current stimulation technology is a non-invasive neuromodulation technology. It is mainly by placing positive and negative electrodes on the scalp and applying a direct current to stimulate the cerebral cortex and increase the excitability of the cerebral cortex, thereby changing the cerebral blood circulation and further improving synaptic performance ${ }^{[9]}$. Studies have found that after 1 month of treatment with transcranial direct current stimulation, the frequency of frozen gait episodes in patients with Parkinson's disease is significantly reduced, and the duration is significantly shortened, and overall it has a good application effect ${ }^{[10]}$. 


\section{Conclusion}

The appearance of freezing gait has a greater impact on the living ability of patients with Parkinson's disease. In order to reduce the burden on the family, effective treatment measures need to be taken as soon as possible. However, conventional drug treatment has no obvious effect, and neuromodulation technology has a good therapeutic effect on the disease, which helps to improve the exercise capacity and quality of life of patients with Parkinson's disease.

\section{References}

[1] Peng Qiaojun, Wang Yufeng, Liu Xin, et al. Research progress in the characteristics and regulation of brain function network of non-motor symptoms in Parkinson's disease. Chinese Journal of Neuroimmunology and Neurology. 2020; 27(5): 407-411.

[2] Wang Xianbin, Tang Hongyan, Li Xingtong, et al. The expression and role of Wnt signaling pathway and neurogenesis and neurodegeneration. Chinese Tissue Engineering Research. 2018; 22(28): 4587-4592.

[3] Zhang Hui, Ma Huiqing, Wang Xiaojuan. Ginkgo biloba extract exerts brain protection on Parkinson's disease rats by activating the Nrf2-ARE signaling pathway. Journal of Shenyang Pharmaceutical University. 2018; 35(8): 675679+695.

[4] Wu Xi, Wang Jiali, Qiu Yiqing, et al. Improving effect of stimulation parameter optimization on temporary non-motor symptoms induced by electrical stimulation after bilateral STN-DBS surgery in Parkinson's disease. Chinese Journal of Clinical Medicine. 2017; 24(6): 845-852.

[5] Shi Kaixuan, Liu Xiaoli, Qiao Decai. The functional connection of the cortex-basal ganglia loop and the neuroplasticity mechanism of PD exercise prevention and treatment. Advances in Physiological Sciences. 2019; 50(1): 25-29.

[6] Lakkappa, Navya, Krishnamurthy, Praveen T., Pandareesh, MD, et al. Soluble epoxide hydrolase inhibitor, APAU, protects dopaminergic neurons against rotenone induced neurotoxicity: Implications for Parkinson's disease. Neurotoxicology. 2019; 70: $135-145$.

[7] Tosato, Marianna, Di Marco, Valerio.Metal Chelation Therapy and Parkinson's Disease: A Critical Review on the Thermodynamics of Complex Formation between Relevant Metal Ions and Promising or Established Drugs. Biomolecules. 2019; 9(7): 269.

[8] Lin Chun, Meng Zhaoxiang, Jin Xing, et al. Clinical research and application of neuromodulation technology in the rehabilitation of Parkinson's disease. Chinese Journal of Physical Medicine and Rehabilitation. 2020; 42(3): $276-279$.

[9] Zheng Yali, Bai Jiahe, Yu Yongpeng. The application progress of neuromodulation technology in the treatment of Parkinson's disease frozen gait. Shandong Medicine. 2020; 60(7): 90-93.

[10] Lebel, Karina,Duval, Christian,Goubault, Etienne, et al. Parkinson's disease patients experiencing peak-dose dyskinesia redistribute involuntary movements throughout their body to improve motor control. Parkinsonism \& Related Disorders. 2019; 64: 312 -314. 